\title{
Spätergebnisse nach Therapie bei Vaginalaplasie
} Somatischer Befund und seelische Verarbeitung

\begin{tabular}{|l|l|l|}
\hline W. & Braendle \\
\hline V. & & Frick-Bruder \\
\hline J. & & Ilse \\
\hline G. & & Bettendorf \\
\hline
\end{tabular}

Abteilung für klinische und experimentelle Endokrinologie der Universitäts-Frauenklinik Hamburg-Eppendorf, BRD

Dr. W. Braendle, Abteilung für klinische und experimentelle Endokrinologie der Universitäts-Frauenklinik, D-2000 Hamburg-Eppendorf (BRD)

Von 47 Patienten mit einer Vaginalaplasie konnten 30 nachuntersucht werden, bei 19 war operativ eine Vaginalplastik durchgeführt worden. Die somatischen Befunde vor der Operation sind in der Tabelle I dargestellt.

Bei 4 Patientinnen, die einen normal grossen Uterus mit Cavum aufwiesen, war der Eingriff wegen einer Hämatometra bzw. eines Hämatokolpos indiziert. In alien anderen Fallen wurde das Vorgehen mit der Patientin und wenn möglich mit ihrem Partner bzw. den Eltern ausführlich besprochen. Die Operationstechnik bestand in einer queren perinea-len Inzision und einer möglichst stumpfen Präparation in die Tiefe, danach Einlage eines Phantoms, wobei in 8 Fallen das Phantom mit Eihaut umlegt wurde. Als postoperative Komplikationen traten in 3 Fallen Blutungen auf, in 1 Fall war eine operative Revision erforderlich. Als häufigste postoperative Komplikation traten Stenosierungen auf, wobei weitere operative Massnahmen im Sinne einer Durchtrennung des Stenoseringes nur in 1 Fall erfolgte. Die Länge der Scheide betrug in diesem Gesamtkollektiv im Mittel 2,3 cm vor der Operation, unmittelbar postoperativ 10,5 cm und bei der Nachuntersuchung (nach 1-14 Jahren) 8,9 cm. Der objektive Operationserfolg wurde entsprechend der Länge und Weite der Vagina in $21 \%$ der Fälle als sehr gut beurteilt, in 32\% als gut, und in 37\% der Fälle war das Resultat weniger gut.

Neben den somatischen gynäkologischen Befunden wurden bei der Nachuntersuchung anhand eines standardisierten Interviews sozialmedizinische und psychologische Parameter miterfasst und diese mit dem operativen Spätergebnis verglichen.

In zwei Dritteln der Fälle war der starke eigene Leidensdruck die wesentliche Motivation zur Operation. In einem Drittel der Fälle überwog der Einfluss von Eltern, Partner oder Ärzten. 66\% der Patientinnen hatten zum Zeitpunkt der Operation eine feste Partner-beziehung, von ihnen nahmen zwei Drittel innerhalb der ersten 4 Monate nach der Operation vaginalen Verkehr auf. Zufrieden mit dem Operationserfolg waren 79\% der Patientinnen nach ihren eigenen Angaben, zufrieden mit dem heutigen Sexualleben 84 \%. Dieser Befund korreliert nicht mit dem objektiven Operationserfolg, von 7 Patientinnen, die einen wenig guten Erfolg aufwiesen, waren nach eigenen Angaben 6 sehr bis recht zufrieden, während bei 3 Patientinnen, die mit dem Erfolg der Operation unzufrieden waren und den Wunsch nach erneuter Operation äusserten, nach dem objektiven Untersuchungsbefund keine Operationsindikation 
Mitteilungen zum 2. Hauptthema 81

Tabelle I. Somatische Befunde vor der Operation

Uterus:solidus bipartitus ( $n=43)$

kleines Cavum $(n=3)$ normal gross mit Cavum uteri $(n=4)$

Tuben/Ovarien: normal ausgebildet

Nieren und ableitende Harnwege: Anomalitäten $(\mathrm{n}=10)$

Skelett: $\quad$ diskrete Anomalien $(n=6)$

Chromosomen: normal weiblicher Karyotyp

bestand. Unter den 11 nicht operierten Patientinnen haben 7 einen festen Partner, 6 sind mit dem heutigen Sexualleben zufrieden. Der objektive Untersuchungsbefund ist sehr unterschiedlich (Scheidenlänge von 1-9 cm). Bei der Hälfte der Patienten war gegenüber dem Vorbefund eine deutliche Zunahme der Länge und Weite der Scheide festzustellen. Alle diese Patientinnen leben in einer festen Partnerschaft und haben regelmässig Kohabi-tationen, dies scheint entscheidend, nicht die Anwendung eines Phantoms, worüber nur von 1 Patientin berichtet wurde. Als wichtigste Ergebnisse der vorliegenden Studie möch-ten wir festhalten:

Der objektive Operationserfolg der Scheidenkorrektur korreliert nicht zwingend mit der subjektiven Zufriedenheit der Patientin und der Zufriedenheit in ihrem Sexualleben.

Die subjektive Zufriedenheit der Patientin mit dem Operationserfolg ist im unter-suchten

Kollektiv weit höher als die Qualität des objektiven Operationserfolges dies erklärt.

Ein uns zufriedenstellender objektiver Operationserfolg korreliert erstaunlicher-weise nicht zwingend mit der subjektiven Zufriedenheit der Patientin bzw. mit der ihres Partners.

Bei vielen der nicht operierten Patientinnen führen allein regelmässige Kohabita-tionen zu einer deutlichen Befundänderung, wobei auch in diesem Kollektiv die Länge und Weite der Scheide nicht mit der subjektiven Zufriedenheit übereinstimmt.

Gynäk. Rdsch. 21 (Suppl. 2): 80-81 (1981) 